\title{
ON THE DISAPPEARANCE OF ISOLATING INTEGRALS \\ IN DYNAMICAL SYSTEMS WITH MORE THAN TWO \\ DEGREES OF FREEDOM
}

\author{
C. FROESCHLÉ and J.-P. SCHEIDECKER \\ Observatoire de Nice, 06300 Nice, France
}

\begin{abstract}
We continue to study the number of isolating integrals in dynamical systems with three and four degrees of freedom, using as models the measure preserving mappings $T$ already introduced in previous papers (Froeschlé, 1972; Froeschlé and Scheidecker, 1973a).

Thus, we use here a new numerical method which enables us to take as indicator of stochasticity the variation with $\boldsymbol{n}$ of the two (respectively three) largest eigenvalues - in absolute magnitude - of the linear tangential mapping $T^{n^{*}}$ of $T^{n}$. This variation appears to be a very good tool for studying the diffusion process which occurs during the disappearance of the isolating integrals, already shown in a previous paper (Froeschlé, 1971). In the case of systems with three degrees of freedom, we define and give an estimation of the diffusion time, and show that the gambler's ruin model is an approximation of this diffusion process.
\end{abstract}

\section{Introduction}

In a previous paper (Froeschlé, 1971), it has been found, using a four-dimensional mapping $T$ as a model problem, that a dynamical system with three degrees of freedom has, in general, either two or zero isolating integrals (beside the usual energy integral).

Let $T$ be a measure preserving mapping of the $(x, y, z, t)$ space over itself defined by:

$$
T\left\{\begin{array}{l}
x_{1}=x_{0}+a_{1} \sin \left(x_{0}+y_{0}\right)+b \sin \left(x_{0}+y_{0}+z_{0}+t_{0}\right), \\
y_{1}=x_{0}+y_{0}, \\
z_{1}=z_{0}+a_{2} \sin \left(z_{0}+t_{0}\right)+b \sin \left(x_{0}+y_{0}+z_{0}+t_{0}\right), \quad(\bmod 2 \pi) \\
t_{1}=z_{0}+t_{0} .
\end{array}\right.
$$

If $b=0$, then this mapping $T$ is the product of two area-preserving mappings $T_{1}$ of $(x, y)$ on itself and $T_{2}$ of $(z, t)$ on itself.

The initial conditions $\left(x_{0}, y_{0}, z_{0}, t_{0}\right)$ are taken such that an invariant curve exists for $T_{1}$ (integrable case) and not for $T_{2}$ (wild or 'ergodic' case).

In this case Froeschlé (1971) has observed that as soon as $b \neq 0$, the value of the isolating integral of $T_{1}$ is subjected to a kind of random walk. This integral slowly disappears by some diffusion process due to the coupling term $b \sin \left(x_{n}+y_{n}+z_{n}+t_{n}\right)$, which produces a quasi-random perturbation of $\left(x_{n}, y_{n}\right)$, as the points $\left(z_{n}, t_{n}\right)$ behave in a quasi-random fashion.

In this paper, we study more precisely numerically this diffusion process and some characteristic parameters of an orbit during this diffusion. One of our tools is the variation of the eigenvalues of the linear tangential mapping $T^{n^{*}}$ of $T^{n}$, which is a good indicator of the stochasticity of an orbit. In particular, we study the character of $C$-system (Arnold and Avez, 1967) of $T$ during the diffusion process.

Hence, we look for the number of eigenvalues which grow exponentially (Froeschlé and Scheidecker, 1973b). 
In Section 2 we study the link between linear tangential mappings and the diffusion process. In Section 3 we define and estimate the diffusion time. In Section 4 we study the variation of this diffusion time with the coupling term and with the initial conditions. In Section 5 we study the case of a dynamical system with four degrees of freedom, i.e. a six-dimensional mapping.

\section{Linear Tangential Mappings and Diffusion Process}

In order to study more precisely the dissolution of the isolating integrals of the discrete dynamical system $T$, we give the topology of the mapping $T_{1}$ in two characteristic cases. This mapping is defined by,

$$
T_{1}\left\{\begin{array}{l}
x_{1}=x_{0}+a_{1} \sin \left(x_{0}+y_{0}\right), \\
y_{1}=x_{0}+y_{0} .
\end{array}(\bmod 2 \pi)\right.
$$

Figures 1 and 2 display typical sets of points for the mapping $T_{1}$. The initial conditions

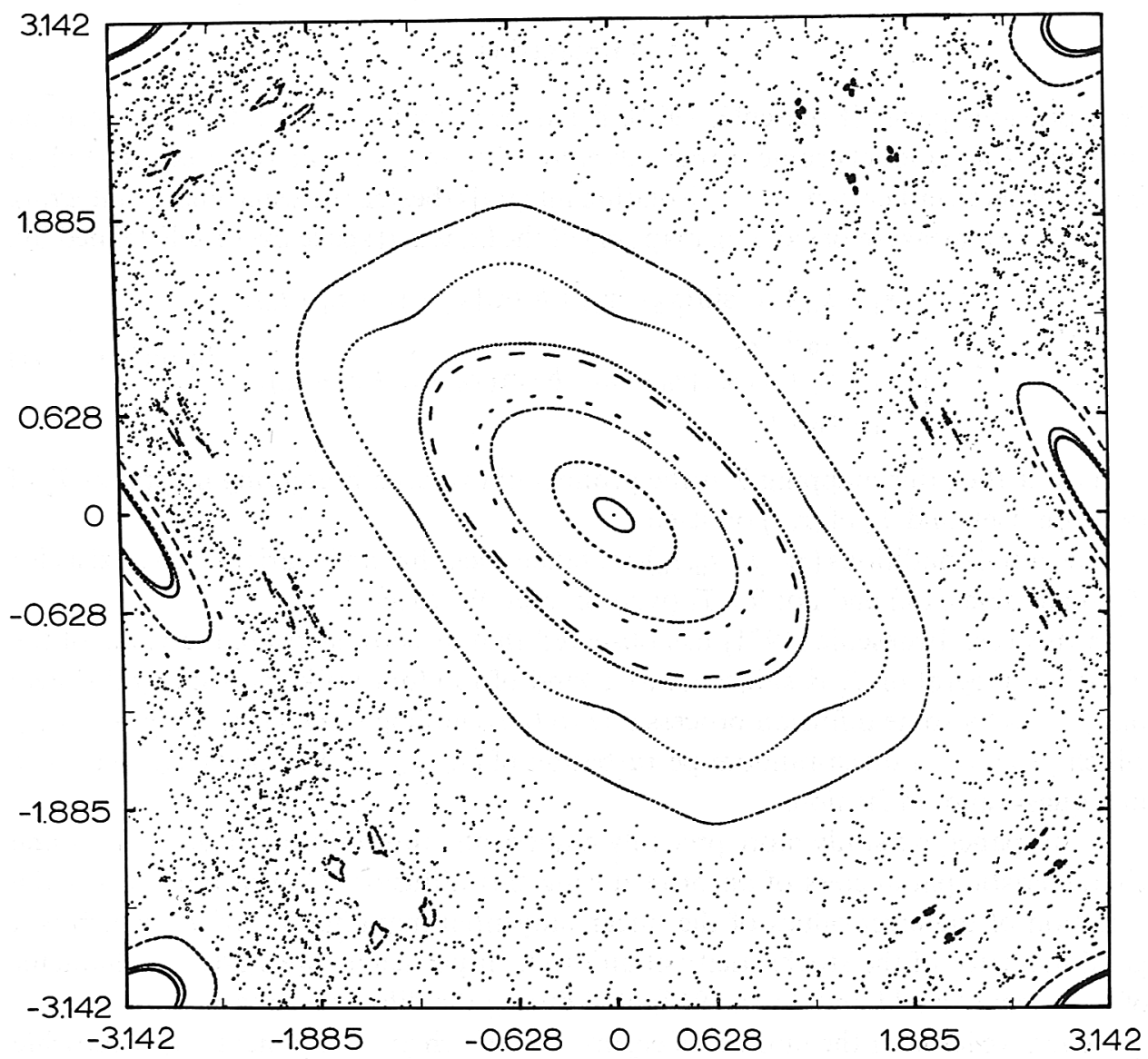

Fig. 1. The mapping $T_{1}$ for $a_{1}=-1.3$. 


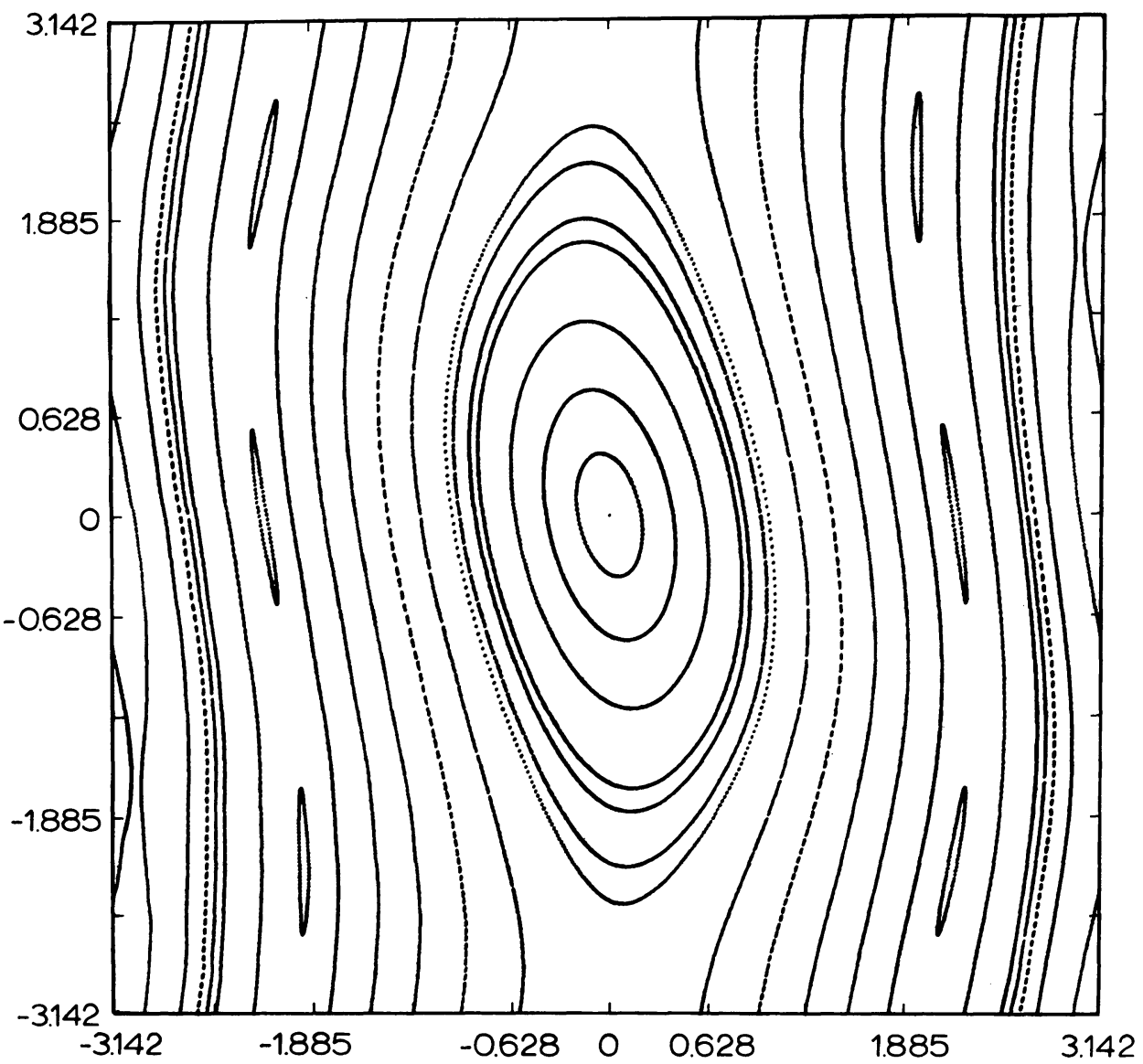

Fig. 2: The mapping $T_{1}$ for $a_{1}=-0.3$.

and values of the parameter $a_{1}$ are presented in Table I. $N$ is the total number of points plotted for each orbit.

Figure 1 exhibits all the characteristics and well-known features of problems with two degrees of freedom, i.e. invariant curves and islands, which correspond to the existence of isolating integrals; and also wild zones, sometimes called 'ergodic', where the points seem to fill a broad region in the plane, and which correspond to the nonexistence of isolating integrals. On the other hand, on Figure 2 isolating integrals seem to exist everywhere: this is a case very close to an integral case. All the points are either on libration curves corresponding to the stable invariant point $(0 ., 0$.$) , or on circulation$ curves corresponding to thè unstable invariant point $(\pi, 0$.).

Now, we study the behavior of the linear tangential mappings during the disappearance of the isolating integrals, i.e. during the diffusion process mentioned in Section 1. Thus, we use the variations with $n$ of the two largest eigenvalues $\lambda_{1}^{n}$ and $\lambda_{2}^{n}$-in absolute magnitude - of the linear tangential mapping $T^{n *}$ of $T^{n}$ (since the characteristic 
TABLE I

Data for Figures 1 and 2

\begin{tabular}{|c|c|c|c|c|}
\hline Figure & $a_{1}$ & $x_{0}$ & $y_{0}$ & $N$ \\
\hline \multirow[t]{21}{*}{1} & -1.3 & 2.8274 & -3.1416 & 700 \\
\hline & & 2,6000 & -3.1416 & 700 \\
\hline & & 2.5133 & -3.1416 & 700 \\
\hline & & 3.0000 & 0 & 700 \\
\hline & & 2.9845 & 0 & 700 \\
\hline & & 2.8274 & 0 & 1000 \\
\hline & & 2.5133 & 0 & 900 \\
\hline & & 2.1991 & 0 & 800 \\
\hline & & 1.8860 & 0 & 800 \\
\hline & & 1.5708 & 0 & 700 \\
\hline & & 1.2566 & 0 & 500 \\
\hline & & 1.0000 & 0 & 400 \\
\hline & & 0.9425 & 0 & 400 \\
\hline & & 0.7000 & 0 & 200 \\
\hline & & 0.6283 & 0 & 300 \\
\hline & & 0.3142 & 0 & 200 \\
\hline & & 0.1000 & 0 & 10 \\
\hline & & 0. & 0 & 3 \\
\hline & & -2.0741 & -1.7318 & 700 \\
\hline & & -1.7880 & -2.3114 & 700 \\
\hline & & 0.1969 & -2.2867 & 700 \\
\hline \multirow[t]{21}{*}{2} & -0.3 & \pm 3.1400 & 0 & 10 \\
\hline & & \pm 3.0000 & 0 & 700 \\
\hline & & \pm 2.8000 & 0 & 700 \\
\hline & & \pm 2.7000 & 0 & 1000 \\
\hline & & \pm 2.6500 & 0 & 400 \\
\hline & & \pm 2.6000 & 0 & 700 \\
\hline & & \pm 2.4000 & 0 & 700 \\
\hline & & \pm 2.2000 & 0 & 700 \\
\hline & & \pm 2.0000 & 0 & 700 \\
\hline & & \pm 1.8000 & 0 & 500 \\
\hline & & \pm 1.6000 & 0 & 500 \\
\hline & & \pm 1.4000 & 0 & 500 \\
\hline & & \pm 1.2000 & 0 & 500 \\
\hline & & \pm 1.0000 & 0 & 500 \\
\hline & & \pm 0.9500 & 0 & 500 \\
\hline & & \pm 0.8500 & 0 & 500 \\
\hline & & \pm 0.8000 & 0 & 500 \\
\hline & & \pm 0.6000 & 0 & 500 \\
\hline & & \pm 0.4000 & 0 & 500 \\
\hline & & \pm 0.2000 & 0 & 200 \\
\hline & & 0. & 0 & 1 \\
\hline
\end{tabular}

equation of the mapping $T$ is reciprocal, the two other eigenvalues are the inverses of the previous ones; hence, they will not be plotted). $\lambda_{1}^{n}$ and $\lambda_{2}^{n}$ have been seen to be characteristics of the stochasticity of an orbit (Froeschlé and Scheidecker, 1973a, b). Numerical results are shown on Figures 3 and 4. Figure 3 displays the variations with 


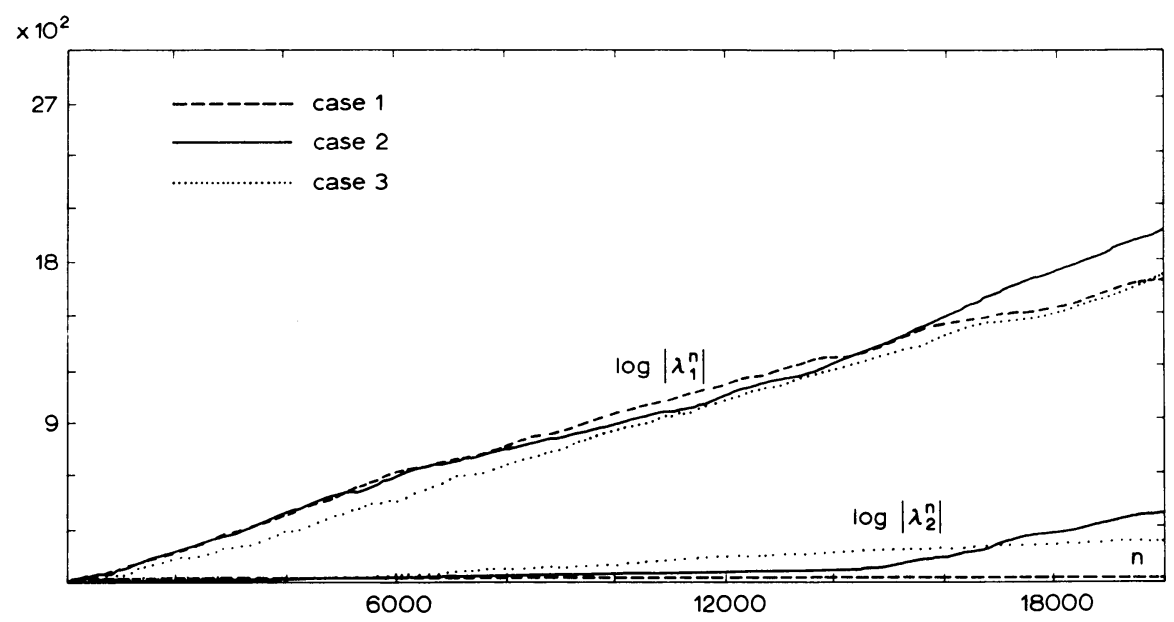

Fig. 3. Eigenvalues of the four dimensional mapping (1). Upper curves: variations of $\log _{10}\left|\lambda_{1}^{n}\right|$ with $n$. Lower curves: variations of $\log _{10}\left|\lambda_{2}^{n}\right|$ with $n$.

$n$ of $\log _{10}\left|\lambda_{1}^{n}\right|$ and $\log _{10}\left|\lambda_{2}^{n}\right|$ in the three following cases:

Case 1: (uncoupled case) $a_{1}=-1.3, a_{2}=-1.3, b=0, x_{0}=0.2, y_{0}=0.2$,

Case 2: $a_{1}=-1.3, a_{2}=-1.3, b=0.05, x_{0}=0.2, y_{0}=0.2, z_{0}=0.5, t_{0}=3$.

$$
z_{0}=0.5, t_{0}=3 \text {. }
$$

Case 3: $a_{1}=-0.3, a_{2}=-1.3, b=0.05, x_{0}=0.2, y_{0}=0.2, z_{0}=0.5, t_{0}=3$.

Remark that in Case 2 (and Case 3), the initial conditions are such that in the corresponding uncoupled case $\left(x_{0}, y_{0}\right)$ is taken in an integrable (or libration) zone for $T_{1}$ and $\left(z_{0}, t_{0}\right)$ in an ergodic zone for $T_{2}$ (cf. Figures 1 and 2 ).

In the uncoupled case (Case 1), the lowest curve shows that one isolating integral does exist because its slope is equal to zero. In the coupled cases (Cases 2 and 3), although a sudden change in the slopes of $\log _{10}\left|\lambda_{2}^{n}\right|$ occurs, when the points $\left(x_{n}, y_{n}\right)$

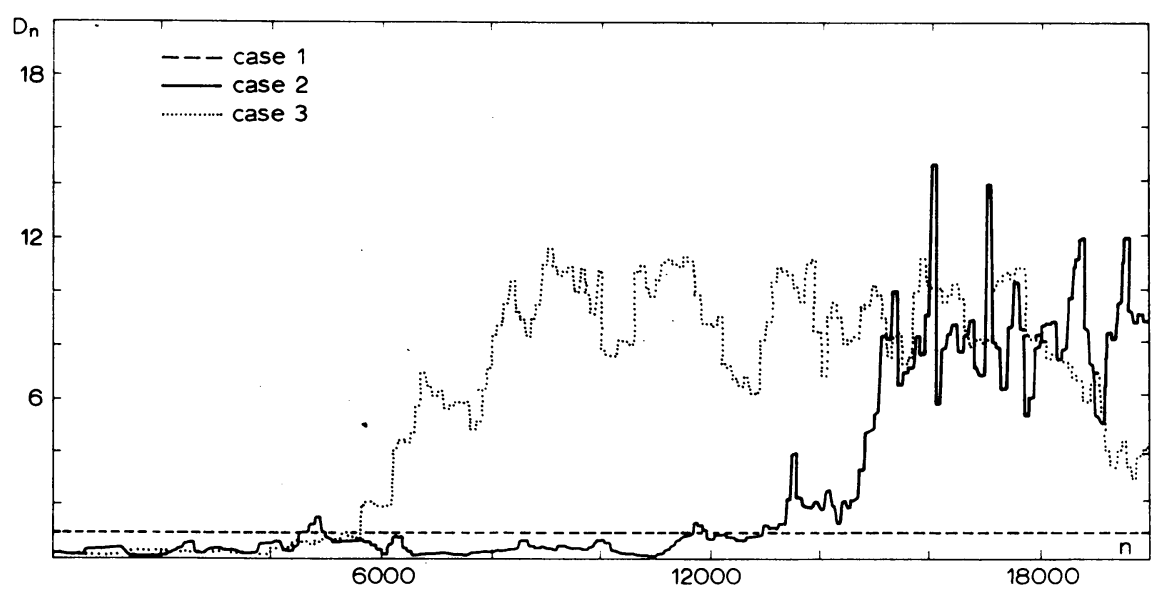

Fig. 4. $D_{n}$, a measure of the dimension of the curves, against $n$. 
have reached either the ergodic zone (Case 2 , for $n=n_{2}=14800$ ) or the circulatory zone (Case 3, for $n=n_{3}=6200$ ), we note that the values of these slopes are always strictly positive. This means that the orbits have an ergodic behavior and that the dynamical system $T$ is close to a $C$-system, even when the diffusion process is still going on. The values of these slopes which are characteristics of the orbits and which change suddenly are related to the topological structure of both two-dimensional mappings $T_{1}$ and $T_{2}$.

This is confirmed by the results displayed on Figure 4, in the same three cases, where $D_{n}$ given by

$$
D_{n}=\left[\sum_{m=n-99}^{m=n}\left(x_{m}^{2}-a_{1}\left(y_{m}^{2}+x_{m} y_{m}\right)\right)\right] / 100
$$

is the measure of the invariant curves of $T_{1}, D_{n}$ being plotted vs $n$, with $n=k \times 100, k$ being a positive integer.

Indeed, we observe in Cases 2 and 3, for the same values of $n$, (Case 2: $n=n_{2}$, Case 3: $n=n_{3}$ ), either a sudden increase of the value of $D_{n}$ (Figure 4) or a sudden change in the slope of $\log _{10}\left|\lambda_{2}^{n}\right|$ (Figure 3). Furthermore, since, in these two cases, we start at the same initial point, the fact that $n_{3}<n_{2}$ may be due to the topology of the curves in Figures 2 and 1. (In Case 3, the libration zone is narrower than in Cases 2. Hence the time necessary for escaping from this zone is smaller in Case 3.)

Moreover, the fact that, in the uncoupled case $\log _{10}\left|\lambda_{2}^{n}\right|$ remains constant, shows clearly that the effects of the rounding errors of the computer are negligible although they could have produced the same effects as the coupling.

\section{Definition and Estimation of the Diffusion Time}

We call 'diffusion time' the number of iterations of the mapping $T$ which are necessary for the point $\left(x_{n}, y_{n}\right)$, starting in the integrable zone, (or the libratory zone) of $T_{1}$, to reach the wild (ergodic) zone (or the circulatory zone) - in other words, the time necessary for the disappearance of isolating integrals. For estimating this time, we use two criteria:

(a) The sudden change in the slope of $\log _{10}\left|\lambda_{2}^{n}\right|$. The estimate of the diffusion time given by this criterion is called $T_{E}$ (eigenvalue criterion).

(b) The sudden change of the value of the measure $D_{n}$ defined in the previous section. As soon as $D_{n}$ is greater or equal to the value $d$, we say that diffusion has occurred. The estimate of the diffusion time using this criterion is called $T_{D}^{d}(4 \leqslant d \leqslant 10)$.

In order to compare $T_{D}^{d}$ and $T_{E}$ we choose at random in the square $(-\pi, \pi) \times(-\pi, \pi)$ points which are tested by the orbits divergence criterion to be either in the invariant curves zone, or in the ergodic zone. The $m$ first ones in the invariant curves zone are taken for $\left(x_{0}, y_{0}\right)$ as well as the $m$ first ones in the ergodic zone, for $\left(z_{0}, t_{0}\right)$. We take as parameters of the mapping $T$ the following values:

$$
b=0.05, \quad a_{1}=a_{2}=-1.3, \quad m=14 .
$$

Figure 5 displays for two fixed values of $d(d=10, d=4)$ and for each initial condition 


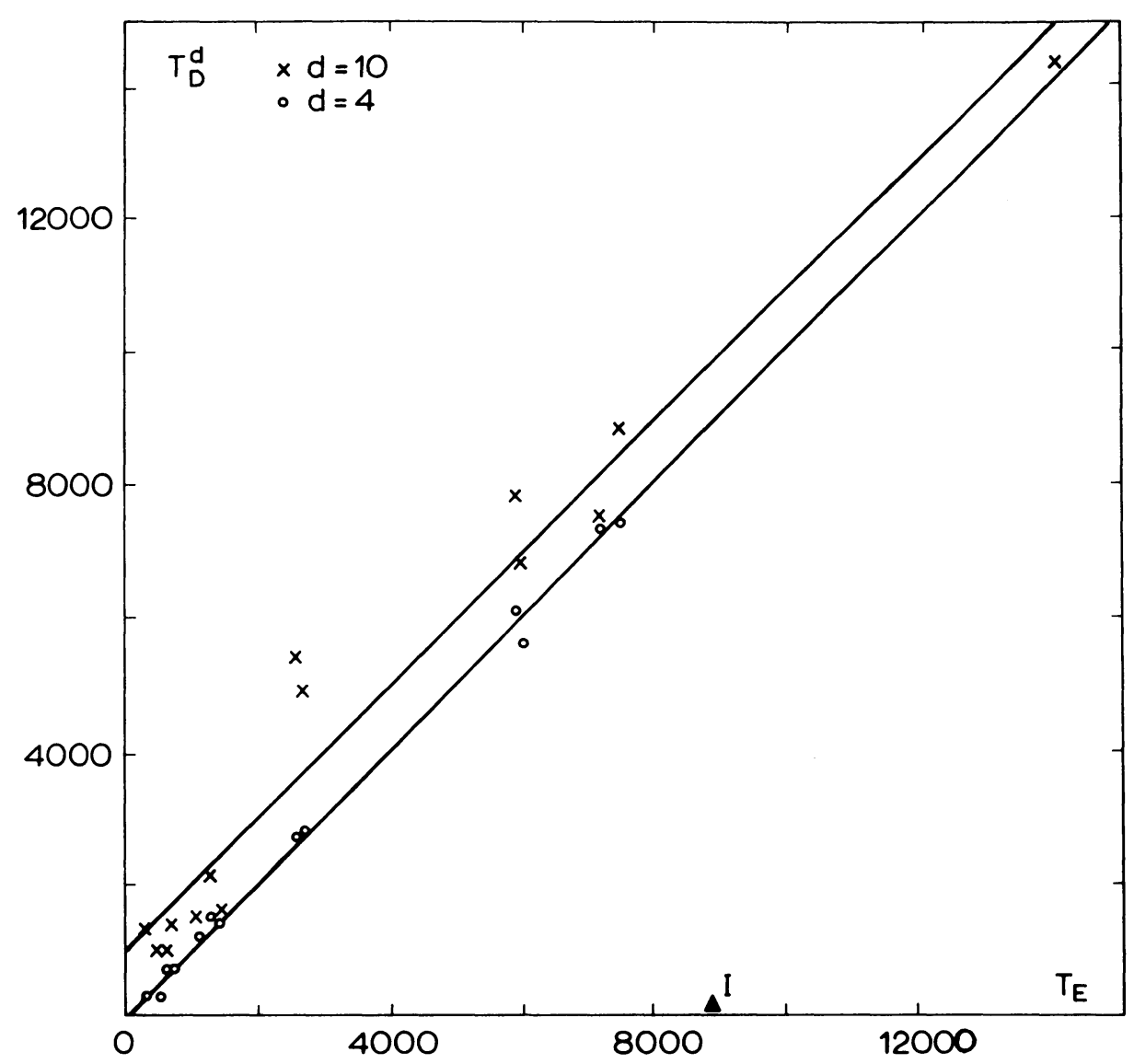

Fig. 5. Comparison of the two diffusion time estimators $T_{E}$ and $T_{D}^{d}$, for $d=10$ and $d=4$.

$\left(x_{0}, y_{0}, z_{0}, t_{0}\right)$ the different values of $T_{D}^{d}$ plotted vs $T_{E}$. The correlation coefficients of the point clouds, and the coefficients $a$ and $b$ of the least square straight line $T_{D}^{d}=a T_{E}+b$ have been computed. The numerical results are given in Table II the straight lines are also shown on Figure 5.

It appears worthwhile to note that for the different values of the parameter $d$, the values of $a$ are close to 1 , with a good precision. This indicates that, for the different experiments, the values of $T_{D}^{d}$ are either overestimated or underestimated by a same amount.

The definition of $D_{n}$ (first-order Birkhoff's approximation) as well as the choice of the different values of $d$ induces errors in the estimation of $T_{D}^{d}$. Moreover, Figure 5 shows also the point $I$ corresponding to integrable initial conditions $\left(x_{0}, y_{0}\right)$ taken in an island (cf. Figure 1), i.e. $\left(x_{0}=-\pi, y_{0}=0\right)$, the point $\left(z_{0}=1.185494, t_{0}=-2.520556\right)$ being taken in an ergodic zone. (Of course, this point has not been taken into account for the computation of the least square straight line.) For this point, $T_{D}^{d}$ is equal to 100 , 
which is the smallest possible value, while $T_{E}$ is rather large. Indeed the use of $D_{n}$ is meaningless, since it is allowed only for the family of invariant curves surrounding the origin.

These facts show us that $T_{E}$ is a more general criterion of diffusion time than $T_{D}^{d}$. However, the computation cost of $T_{E}$ is about 20 times as large as the computation

TABLE II

Values of the correlation coefficients and coefficients of the least square straight line $T_{D}^{d}=a T_{E}+b$ of the point clouds for different values of $d$

\begin{tabular}{rllc}
\hline$d$ & $\begin{array}{l}\text { Correlation } \\
\text { coefficient }\end{array}$ & $a$ & $b$ \\
\hline 4. & 0.99905 & 1.0096 & 0.04989 \\
6. & 0.99751 & 0.9915 & 195.765 \\
8. & 0.99706 & 1.011 & 360.719 \\
10. & 0.9795 & 0.9912 & 1017.92
\end{tabular}

cost of $T_{D}^{d}$. This is due to the fact that it is necessary to use a special programme for computing the second eigenvalue (Froeschlé and Scheidecker, 1973b). Hence, in the following we shall use $T_{D}^{d}$ as diffusion time estimator since we can avoid cases such as point $I$ on Figure 5, using the topology of the curves on Figures 1 and 2. Of course, $T_{E}$ has been used to check some results given by this last method.

\section{Variation of the Diffusion Time with the Coupling Term $b$ and with the Distance of the Initial Point to the Origin}

We intend to estimate the diffusion time as a function of the coupling parameter $b$ and as a function of the generalized distance of the initial point to the origin.

We take the gambler's ruin model as an approximation of the diffusion process, since the problem is reduced to the study of the jumps of $\left(x_{n}, y_{n}\right)$ from one elliptic curve to another, up to the ergodic zone, considered as an absorbing barrier. Indeed, the family of invariant curves surrounding the origin can be taken, in a first-order approximation, as a continuous elliptic family (cf. Figures 1 and 2).

In order to handle the problem more easily, we take a discrete family of homofocal ellipses as a model. The iterated point is supposed to jump from one ellipse to the next one. In fact, this problem reduces itself to a one-dimensional gambler's ruin problem, since the equations of the family of homofocal ellipses are given by:

$$
x^{2}-a_{1}\left(y^{2}+x y\right)=C_{k}, \quad k=0, \ldots, k_{1},
$$

$k_{1}$ being the value corresponding to the largest ellipse, that is to say, to the absorbing barrier. The point $\left(x_{n}, y_{n}\right)$ starts from the initial condition $\left(x_{0}, y_{0}\right)$ belonging to the ellipse $C_{k}$, and moves a step at random at each iteration, backward or forward, to $C_{k+1}$ or to $C_{k-1}$. 
We put $X^{2}=C_{k}$. $X$ can be interpreted as one of the two values of the intersection of the ellipse $C_{k}$ with the $x$-axis. We call $\varepsilon$ the increase $\Delta X$, taken as a constant, when we jump from one ellipse to the next one.

Let $N(X)$ be the expected number of iterations, which are necessary for reaching the absorbing barrier, when starting on the ellipse $C_{k}$, at the generalized distance $X$ from the origin.

We use the well-known formula (Feller, 1971)

$$
N(X)=\frac{N(X+\varepsilon)+N(X-\varepsilon)}{2}+1 \text {. }
$$

The boundary conditions are:

$$
N\left(+C_{k_{1}}^{1 / 2}\right)=0, \quad N\left(-C_{k_{1}}^{1 / 2}\right)=0 .
$$

Taking a second-order Taylor development, we get:

$$
\mathrm{d}^{2} N(X) / \mathrm{d} X^{2} \simeq-2 / \varepsilon^{2} .
$$

Hence, by integration:

$$
N(X) \simeq-\left(2 / \varepsilon^{2}\right) X^{2}+A X+B .
$$

Using (6), we get:

$$
N(X) \simeq 2\left(C_{k_{1}}-X^{2}\right) / \varepsilon^{2} .
$$

\subsection{VARIATIONS WITH THE COUPLING TERM $b$}

We use Equation (9) with $X$ equal to a fixed value $\bar{X}$, and take as initial conditions $x_{0}=y_{0}=0.5$. Hence, by (4), we compute $\bar{X}=0.9486$; from the measure of $C_{k_{1}}^{1 / 2}$ taken on Figure 1 we get the approximate value $C_{k_{1}}=2.89$.

In a first approximation, we consider the length of the step to be $\varepsilon$, and to be proportional to the coupling term $b$. Therefore, we have plotted the variation of $T_{D}^{9} b^{2}$ vs $b$ on Figure 6 ( $b$ takes values from 0.01 to 0.2 ). The straight line which is displayed is the average of the values of $T_{D}^{9} b^{2}$. (This value is equal to 7.77.)

The points displayed by Figure 6 are obtained by using the computed values $T_{D}^{9}$ of the diffusion time $N(X)$ : each of these values is itself the average of 25 computed values, corresponding to initial conditions $\left(x_{1}^{\theta}, y_{1}^{\theta}, z_{1}^{\theta}, t_{1}^{\theta}\right)$ surrounding the point $\left(x_{0}, y_{0}, z_{0}, t_{0}\right)$, at random:

$$
\begin{aligned}
x_{1}^{\theta} & =x_{0}, \\
y_{1}^{\theta} & =y_{0}, \\
z_{1}^{\theta} & =z_{0}+r_{0} \cos \theta, \\
t_{1}^{\theta} & =t_{0}+r_{0} \sin \theta,
\end{aligned}
$$

where $r_{0}=10^{-4}$ and $\theta$ is chosen at random between $-\pi$ and $+\pi$. Such a method tends to give us more precision in the result. 


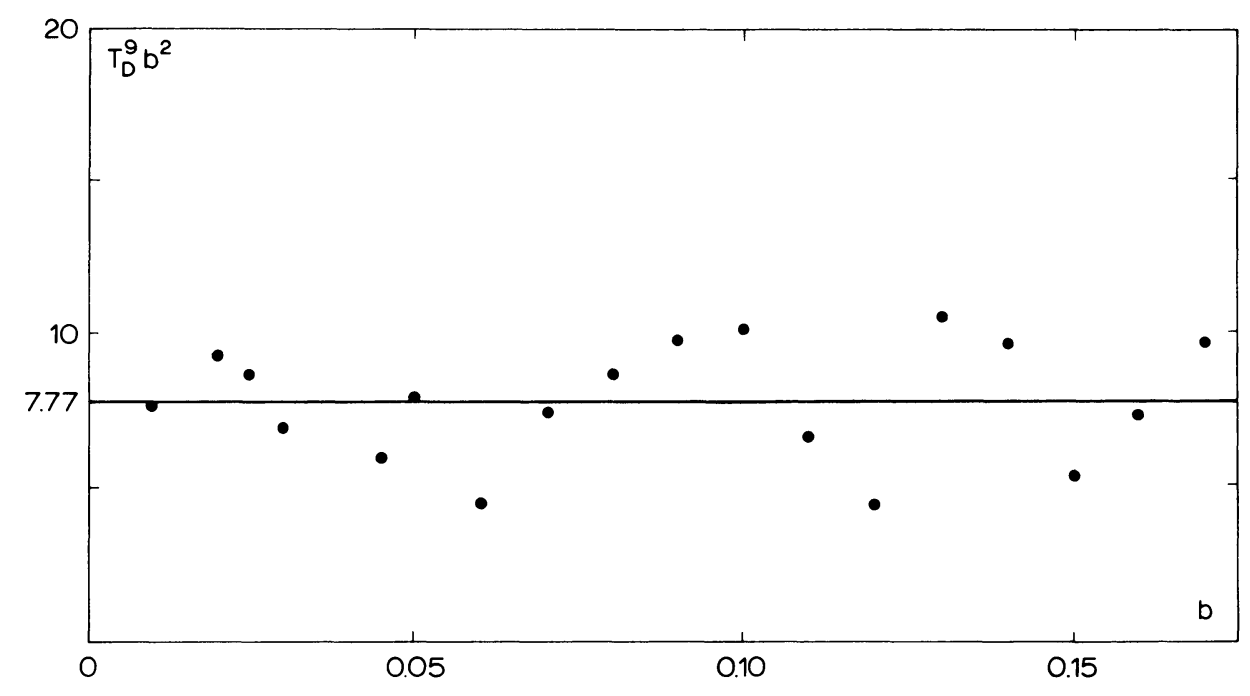

Fig. 6. Verification of the law $N(X) \varepsilon^{2} \simeq$ constant, for a given $X$.

Considering the crude approximations which have been made for obtaining Equation (9) the results are in rather good agreement with the gambler's ruin model.

From the preceding numerical results (mean value of $T_{D}^{9} b^{2}$, values of $\bar{X}$ and $C_{k_{1}}^{1 / 2}$ ), using Equation (9) we get:

$$
\varepsilon^{2} / b^{2} \cong \frac{1}{2}
$$

\subsection{VARIATIONS WITH THE INITIAL CONDITION $X$}

We take as fixed value of the coupling term: $b=0.05$. From (9), we get:

$$
\varepsilon^{2} N(X)+2 X^{2} \simeq 2 C_{k_{1}},
$$

where

$$
X^{2}=x_{0}^{2}-a_{1}\left(y_{0}^{2}+x_{0} y_{0}\right), \quad a_{1}=-1.3 .
$$

And from (11):

$$
\varepsilon^{2} \simeq 12.5 \times 10^{-4} .
$$

Thus, we plot the values of $T_{D}^{9} b^{2}+4 X^{2}$ vs $X$ on Figure 7 , since the $T_{D}^{9}$ are the experimental values of $N(X)$. Each value of $T_{D}^{9}$ is computed in the same way as previously (average of 25 random points). The straight line represents the average of the values of $T_{D}^{9} b^{2}+4 X^{2}$, which is found to be equal to 11.48 , hence, to be very close to $4 C_{k_{1}}=11.56$.

Also, in this case, the gambler's ruin model seems to be a good approximation of the diffusion problem. 


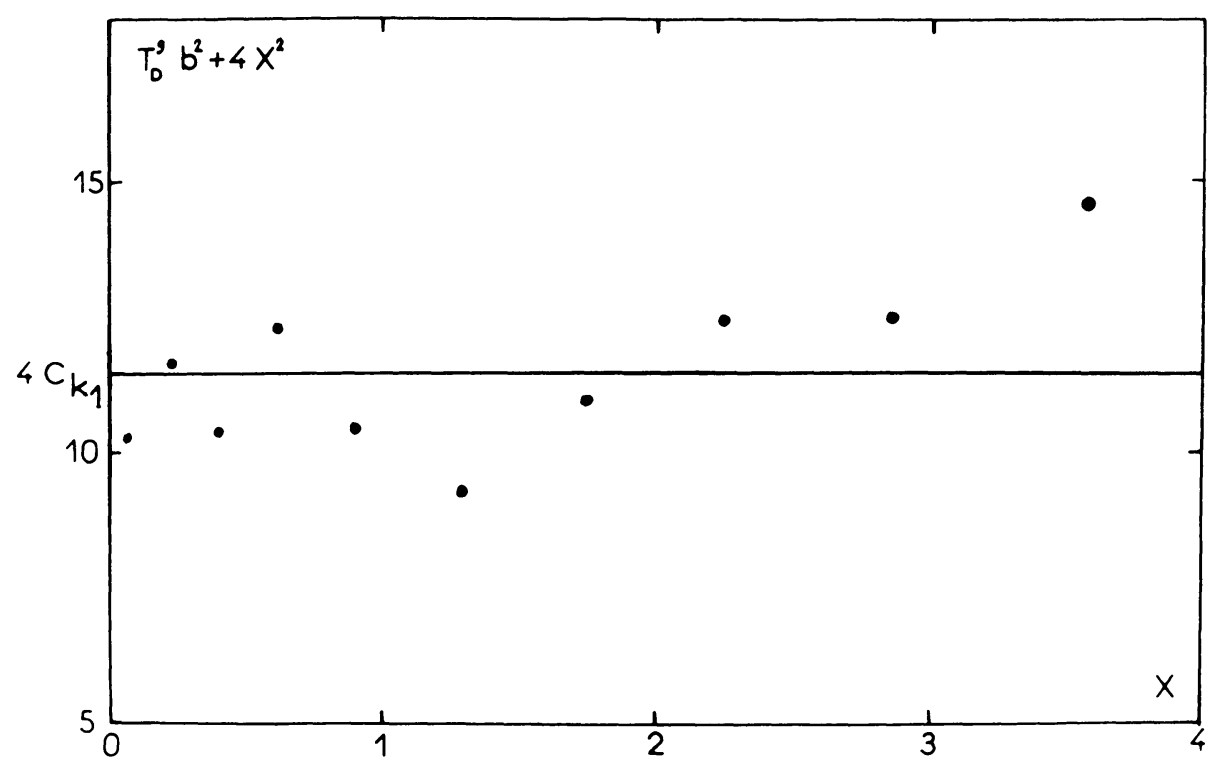

Fig. 7. Verification of the law $N(X) \varepsilon^{2}+2 X^{2} \simeq$ constant, for a given $\varepsilon$.

\section{The Case of Dynamical Systems with Four Degrees of Freedom}

The results given by Froeschle (1971), and the previous sections in this paper suggest that, apart from particular cases such as $b=0$ for the present model, dynamical systems with three degrees of freedom have in general either two or zero isolating integrals, beside the usual energy integral. A similar effect probably exists in systems with more than three degrees of freedom.

We generalize the mapping $T$ to the following six-dimensional mapping $T_{6}$, that is to say, to a dynamical system with four degrees of freedom:

$T_{6}\left\{\begin{array}{l}x_{1}=x_{0}+a_{1} \sin \left(x_{0}+y_{0}\right)+b \sin \left(x_{0}+y_{0}+z_{0}+t_{0}+u_{0}+v_{0}\right), \\ y_{1}=x_{0}+y_{0}, \\ z_{1}=z_{0}+a_{2} \sin \left(z_{0}+t_{0}\right)+b \sin \left(x_{0}+y_{0}+z_{0}+t_{0}+u_{0}+v_{0}\right), \quad(\bmod 2 \pi) \\ t_{1}=z_{0}+t_{0}, \\ u_{1}=u_{0}+a_{3} \sin \left(u_{0}+v_{0}\right)+b \sin \left(x_{0}+y_{0}+z_{0}+t_{0}+u_{0}+v_{0}\right), \\ v_{1}=u_{0}+v_{0} .\end{array}\right.$

If $b=0$, this mapping $T_{6}$ is the product of three two-dimensional area preserving mappings: $T_{1}$ of $(x, y)$ on itself, $T_{2}$ of $(z, t)$ on itself, and $T_{3}$ of $(u, v)$ on itself, and as in previous sections, we take initial conditions such that for $b=0$, we have either $N=0$, 1,2 or 3 isolating integrals.

\section{1. $N=0$}

This corresponds to a purely ergodic case. Figure 8 displays the variation vs $n$ of the 
logs of the three largest eigenvalues, $\lambda_{1}^{n}, \lambda_{2}^{n}, \lambda_{3}^{n}$ (in decreasing order of absolute magnitude) of $T^{n *}$.

The initial conditions are: $x_{0}=2.0, y_{0}=0.0, z_{0}=2.1, t_{0}=0.0, u_{0}=2.2, v_{0}=0$.

The values of parameters are: $a_{1}=a_{2}=a_{3}=-1.3, b=0.050$.

5.2. $N=1,2$

Here, we have, for the uncoupled case, one or two isolating integrals. Figures 9 and 10 show the same process as in the case of three degrees of freedom.

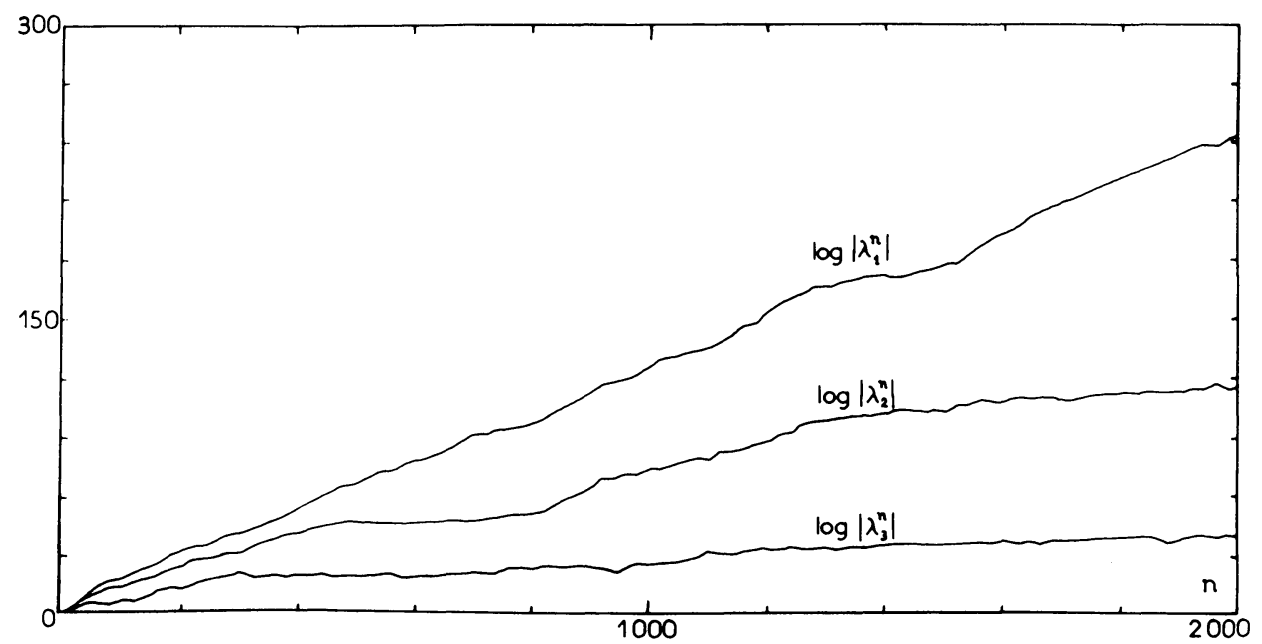

Fig. 8. Eigenvalues of the six-dimensional mapping (14): variations of $\log _{10}\left|\lambda_{1}^{n}\right|, \log _{10}\left|\lambda_{2}^{n}\right|, \log _{10}\left|\lambda_{3}^{n}\right|$ with $n$, when no isolating integrals exist.

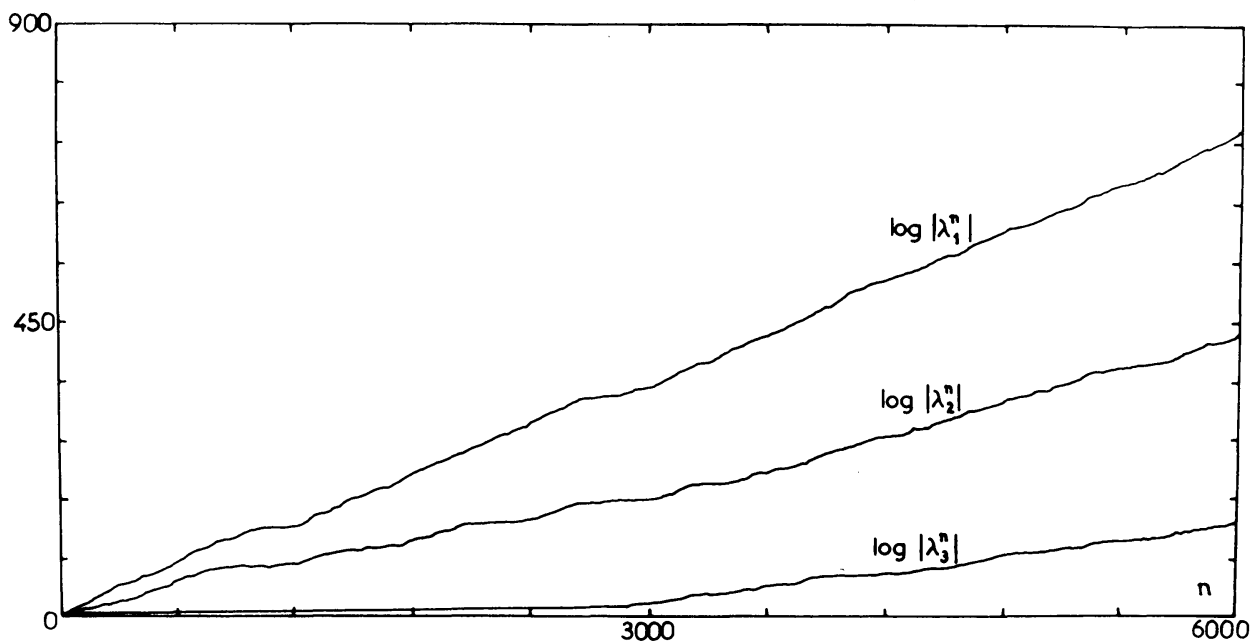

Fig. 9. Variations of $\log _{10}\left|\lambda_{1}^{n}\right|, \log _{10}\left|\lambda_{2}^{n}\right|, \log _{10}\left|\lambda_{3}^{n}\right|$ with $n$, when, for the corresponding uncoupled case, one isolating integral exists. 
Initial conditions for Figure 9 are: $x_{0}=0.5, y_{0}=0.5, z_{0}=0.5, t_{0}=3.0, u_{0}=0.5, v_{0}=3.1$. Initial conditions for Figure 10 are: $x_{0}=0.5, y_{0}=0.5, z_{0}=0.1, t_{0}=0.1, u_{0}=0.5$, $v_{0}=3.0$.

Parameters are: $a_{1}=a_{2}=a_{3}=-1.3 ; b=0.052$ for both cases. The value of $b$ has been slightly increased in order to decrease the number of iterations necessary to reach the ergodic zone.

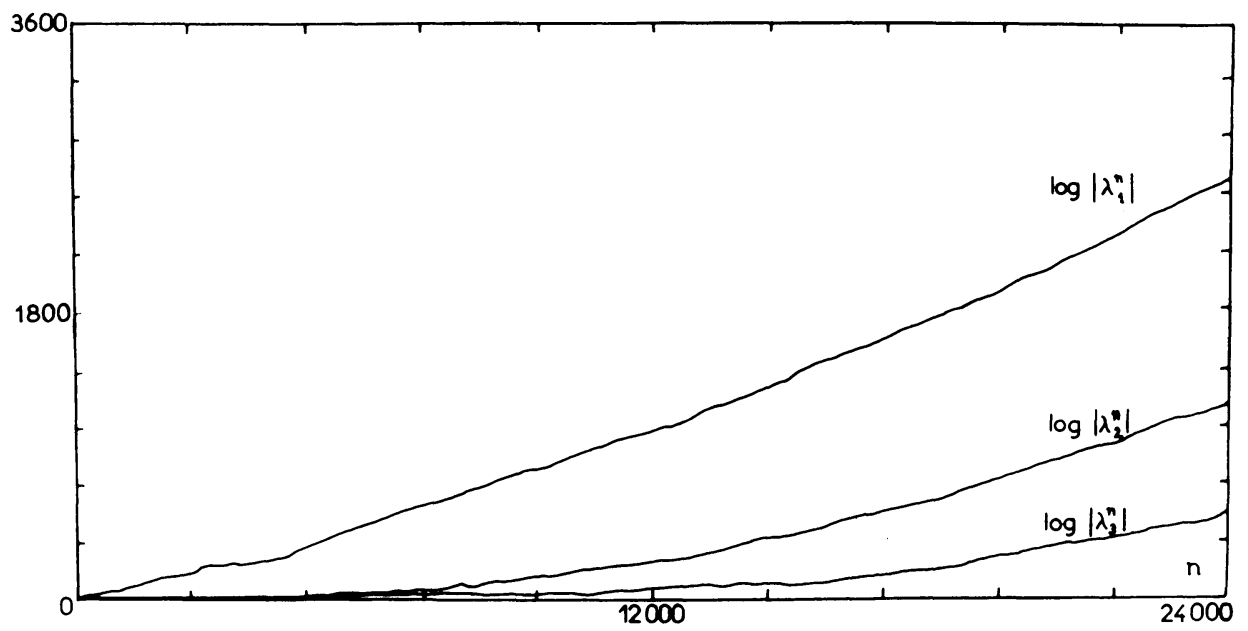

Fig. 10. Variations of $\log _{10}\left|\lambda_{1}^{n}\right|, \log _{10}\left|\lambda_{2}^{n}\right|, \log _{10}\left|\lambda_{3}^{n}\right|$, when, for the corresponding uncoupled case, two isolating integrals exist.

These figures show a sudden change in the slopes of the logs of the third eigenvalue (respectively 2 nd and 3rd eigenvalues), and we note also that the values of these slopes are always strictly positive. Hence, the orbits have an ergodic behavior, and $T_{6}$ is close to a $C$-system.

\section{3. $N=3$}

It corresponds to a purely integrable case. In this case the slopes of the logs of the three eigenvalues remain equal to zero. Thus, these results confirm the phenomena already observed in the case of three degrees of freedom.

\section{Conclusions}

The results obtained in particular by means of the study of the variation with $n$ of the logs of the largest eigenvalues of the linear tangential mapping $T^{n^{*}}$ of $T^{n}$ confirm those given by Froeschlé (1971).

The character of $C$-system of the orbits has been shown to appear even during the diffusion process occuring when the isolating integrals disappear.

Furthermore, it has been shown that the gambler's ruin model is a rather good approximation of the diffusion process. 
Finally, using a six-dimensional mapping, we have confirmed numerically the conjecture given by Froeschlé(1971) that a dynamical system with $n$ degrees of freedom has in general either $n-1$ or 0 isolating integrals, beside the usual energy integral.

\section{Acknowledgement}

We have the pleasure of thanking Dr Michel Hénon for helpful discussions during this work.

\section{References}

Arnold, V. I. and Avez, A.: 1967, Problèmes ergodiques de la mécanique classique, Gauthier-Villars, Paris. Feller, W.: 1971, An Introduction to Probability Theory and Its Applications, Vol. 1, Wiley, New York. Froeschlé, C.: 1971, Astrophys. Space Sci. 14, 110.

Froeschlé, C.: 1972, Astron. Astrophys. 16, 172.

Froeschlé, C. and Scheidecker, J.-P.: 1973a, Astron. Astrophys. 22, 431.

Froeschlé, C. and Scheidecker, J.-P.: 1973b, J. Comput. Phys. 11-3, 423.

\section{DISCUSSION}

G. Contopoulos: You have taken the point $\left(x_{0}, y_{0}\right)$ in the isolating region and $\left(t_{0}, z_{0}\right)$ in the ergodic region. I would like to ask: (a) What happens if $\left(t_{0}, z_{0}\right)$ is in the isolating region also? Did you find any indication of Arnold diffusion? (b) What happens at the transition region, as $\left(t_{0}, z_{0}\right)$ goes from the isolating to the ergodic region?

C. Froeschlé: (a) If $\left(z_{0}, t_{0}\right)$ is also in the isolating region we have found that if the coupling term is not large the points remain on a two-dimensional manifold of the four-dimensional space $\left(x_{0}, y_{0}, z_{0}, t_{0}\right)$. We have not found any indication of Arnold diffusion. (b) This case has not been studied. We start either in the isolating region or in the ergodic region.

J. Moser: What is the order of time considered in your numerical experiments? I want to comment that recent calculation by a physicist at Brookhaven has indicated that for about $10^{5}$ periods one had satisfactory bounds for the solution although after that to about $10^{7}$ periods definitive deterioration was observed.

C. Froeschlé: The order of magnitude was about $2 \times 10^{5}$ periods.

G. Contopoulos: I would like to report on some recent work by a group of theoretical physicists in Milan, Italy, under Prof. Scotti. They studied the motion of $N$ (non-linear) coupled oscillators $(N=10)$. At the same time they calculated analytically $N$ formal integrals of motion, using a computer programme that I developed a few years ago. They found that as the energy (or the coupling constant) increases there is a threshold, above which the motion becomes ergodic. This threshold seems to remain finite (different from zero) as the number of degrees of freedom increases. Thus they conjecture that, in general, as $N$ becomes large, the motion does not become ergodic if the coupling is below certain limit. 